\title{
Development of new baked bricks based on clay and sawdust
}

\author{
Omrane Benjeddou ${ }^{1,2}$, Chokri Soussi ${ }^{1,3}$, Mohamed Amine Khadimallah ${ }^{4,5}$, Rayed Alyousef ${ }^{4}$, Malek Jedidi ${ }^{1,2}$ \\ ${ }^{1}$ Higher Institute of Technological Studies of Sfax, Department of Civil Engineering, Sfax, Tunisia \\ ${ }^{2}$ University of Tunis El Manar, National Engineering School of Tunis, Civil Engineering Laboratory, Tunis, Tunisia \\ ${ }^{3}$ University of Sfax, Faculty of science of Sfax, Georessources, Materials, Environment and Global Change Laboratory, \\ Sfax, Tunisia. \\ ${ }^{4}$ Prince Sattam bin Abdulaziz University, College of Engineering, Civil Engineering Department, Alkharj, Saudi Arabia \\ ${ }^{5}$ University of Carthage, Polytechnic School of Tunisia, Laboratory of systems and applied mechanics, Tunis, Tunisia
}

\begin{abstract}
Current research in the field of building materials is oriented towards the recovery of waste to ensure the lightening of certain building elements and energy saving. The objective of this study is to produce a new bricks by adding sawdust to clay mixtures. The first part of this experimental work consists on detremining the proper manufacturing process and cooking diagram for these new bricks. In the second part, the effect of the maximum cooking temperature, the firing phase time's of the bricks and the sawdust prportion on the physical and mechanical properties of the new bricks have been studied. The studied properties are the absorption ratio, the unit weight and the compressive strength. The results showed that the addition of an increasing amount of sawdust tends to modify the physical and mechanical properties of the cooked samples. The presence of sawdust reduces the number of connections between the clay grains and produces a lighter, more absorbent and less resistant material. Finally, the results showed that the manufacture of bricks based on sawdust is possible.
\end{abstract}

Résumé. La recherche actuelle dans le domaine des matériaux de construction est orientée vers la valorisation des déchets pour assurer l'allégement de certains éléments de construction et une économie d'énergie. L'objectif de cette étude est de produire des nouvelles briques d'argile plus légères que celles qui sont actuellement sur le marché, en ajoutant des sciures de bois aux mélanges d'argile. Plusieurs paramètres ont été étudiés ainsi que leur effet sur les modifications des propriétés physiques et mécaniques tels que le taux d'absorption, la température maximale de cuisson, la proportion des sciures et la durée de la phase de cuisson de ces briques. Un processus de fabrication, de séchage et de cuisson a été adapté au nouveau produit. Les résultats ont montré que l'addition d'une quantité croissante de sciures tend à modifier sensiblement les propriétés physiques et mécaniques des échantillons cuits. La présence de sciures réduit le nombre de connections entre les grains d'argile et produit un matériau plus léger, plus absorbant et moins résistant. Enfin, les résultats ont montré aussi que la confection des briques à base des sciures de bois est possible.

\section{Introduction}

La recherche actuelle dans le domaine des matériaux de construction est orientée vers la valorisation des déchets pour assurer d'une part, la pérennité des granulats naturels conventionnels et l'allégement de certains éléments de construction et d'autre part, une économie d'énergie.

L'industrie du bois génère des quantités énormes de déchets (rebus de bois, copeaux et sciures) par an dans le monde. Différentes filières de valorisation de ces déchets existent mais restent très nocives pour l'environnement. Un nouvel enjeu est de trouver des voies et moyens pour une valorisation durable de ces sous-produits. La valorisation surtout des sciures de bois dans la construction représente une solution durable.

L'utilisation des sciures de bois dans l'industrie des briques peut non seulement permettre leur valorisation de façon importante et durable mais aussi permettre la réduction de l'impact environnemental de cette industrie.

Un des objectifs de ce projet est d'élaborer de nouveaux matériaux de construction à base de sciures de bois compétitifs et concurrents des matériaux de construction classiques connus. Il s'agit de développer un nouveau produit : ce sont des briques à base d'argile et de sciures de bois. Ce matériau est obtenu en mélangeant l'argile, la sciure de bois, et l'eau.

Nous avons, à partir d'une série d'essais réalisés au laboratoire Génie Civil de L'Institut Supérieur des Etudes Technologiques de Sfax, sur la pâte d'argile-sciure de bois, déterminé la teneur optimale d'eau et le volume de pâte à utiliser pour bien remplir les moules de la presse de compactage afin d'avoir des briques bien consolidées.

Pour caractériser l'effet de l'utilisation de la sciure de bois sur la qualité des briques, nous avons soumis les briques réalisées, et ce après un cycle de séchage et de 
cuisson bien déterminé, à des essais tels que l'essai de la résistance à la compression, le comportement hydrique (absorption et tenue dans l'eau).

\section{Le protocole expérimental} parties :

L'étude en laboratoire se subdivise en plusieurs

$>$ La première partie porte sur la caractérisation des trois échantillons de matières premières prélevées.

$>$ La deuxième partie a pour objectif de se familiariser avec le processus de fabrication et de déterminer la méthode optimale de cuisson des nouvelles briques.

$>$ La troisième partie analyse les modifications subies par les briques d'argile lorsque des sciures de bois sont ajoutées aux mélanges.

$>$ La dernière partie est consacrée à l'étude de l'effet de la variation du ratio de sciures de bois sur les différentes propriétés des briques.

\section{Identification des matériaux utilisés}

\subsection{Identification de l'argile}

L'argile est broyée à l'état sec à l'aide d'un concasseur afin de l'homogénéiser.

\subsubsection{Teneur en matières organiques de l'argile}

L'essai consiste à déterminer la perte de masse d'un échantillon d'argile sèche dans un four à une température de $450{ }^{\circ} \mathrm{C}$. La teneur en matières organiques $\mathrm{C}_{\mathrm{MOC}}=1.2$ $<3$ : L'argile est classée non organique.

\subsubsection{Poids volumique des grains solides}

La masse volumique des grains solides de l'argile est $\rho s=2.65 \mathrm{~g} / \mathrm{cm}^{3}$. Cette valeur de la masse volumique prouve que l'argile est dans la gamme des sols inorganiques.

\subsubsection{Limites d'Atterberg}

Le tableau 1 présente les limites d'Atterberg, ainsi que l'indice de plasticité, l'indice de liquidité et l'indice de consistance pour l'argile testée.

Tableau 1. Les limites d'Atterberg de l'argile utilisée

\begin{tabular}{|c|c|c|c|c|c|c|}
\hline 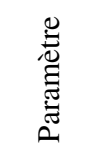 & 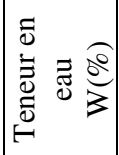 & 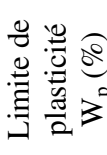 & 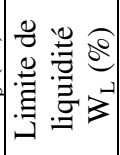 & 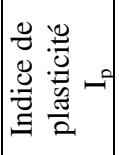 & 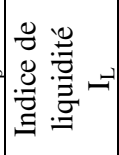 & 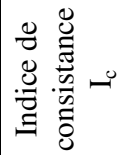 \\
\hline Valeur & 0 & 25 & 52 & 27 & 1 & 2 \\
\hline
\end{tabular}

Les résultats montrent que l'échantillon de l'argile a une valeur d'indice de plasticité de 27 , ceci permet de les classer parmi les sols très plastiques. Par ailleurs, la limite de plasticité obtenue $\mathrm{Wp}=25$ donne une valeur approximative sur la quantité d'eau nécessaire lors de façonnage des briques (extrusion),

\subsubsection{Analyse granulométrique}

Les résultats de l'analyse granulométrique par sédimentation (Norme NF P 94-057) montre que notre argile est constituée à peu près de :

- $40 \%$ de limon fin

- $20 \%$ de limon moyen

- $12 \%$ de limon gros

- $18 \%$ sable $0 / 2$

Ceci nous permet de juger à priori que l'ajout du sable comme dégraissant n'est pas nécessaire.

\subsubsection{Essai au bleu de méthylène et surface spécifique}

Les valeurs du bleu de méthylène et de la surface spécifique de l'argile sont respectivement $\mathrm{V}_{\mathrm{BS}}=1.15$ et $\mathrm{SSB}=28 \mathrm{~m}^{2} / \mathrm{g}$. Ces valeurs prouvent que l'argile est composée majoritairement de limon, mais le calcul de l'activité montre qu'il s'agit d'une argile très active.

\subsection{Identification des sciures}

\subsubsection{Préparation des sciures:}

Les sciures de bois sont fabriquées au laboratoire.
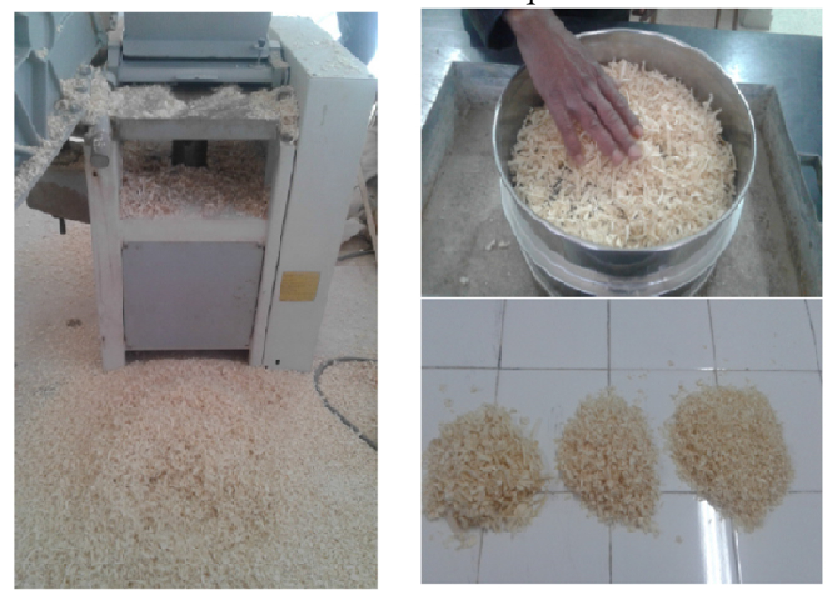

Fig. 1. Préparation des sciures de bois au laboratoire

\subsubsection{Composition chimique}

Quelques soient les essences d'arbres considérées, la composition chimique du bois est approximativement la même : $50 \%$ de carbone, $6 \%$ d'hydrogène et $44 \%$ d'oxygène. La valeur calorifique, par kilogramme, est aussi constante pour toutes les espèces, soit environ 8370 KJ. Quant aux températures de combustion, elles s'établissent entre $300^{\circ} \mathrm{C}$ et $500^{\circ} \mathrm{C}$, la majeure partie des réactions exothermiques se terminent à des températures voisines de $500^{\circ} \mathrm{C}$.

Suite à ces informations très intéressantes, les sciures de bois quelque soit leur origine se comportent de la même manière lors de l'opération de cuisson.

Le caractère fortement hygroscopique des sciures lorsqu'elles sont introduites dans la pate d'argile, fait qu'elles absorbent une partie de l'eau, c'est pourquoi, il 
faut prendre en compte ce paramètre lors de la préparation de la pate.

\subsubsection{Détermination des masses volumiques apparente et absolue :}

La masse volumique apparente et la masse volumique absolue des sciures en bois sont respectivement égales à $\rho a=0.060 \mathrm{~g} / \mathrm{cm}^{3}$ et $\rho s=0.50$ $\mathrm{g} / \mathrm{cm}^{3}$.

\section{Procédé de préparation des échantillons}

Les étapes du procédé de préparation des échantillons sont les suivantes :

\subsection{Malaxage à sec du mélange}

On commence par ajouter les sciures à l'argile. Puis on mélange manuellement ces deux composantes jusqu'à leur homogénéisation. Enfin, ce mélange est malaxé mécaniquement dans un malaxeur électrique pendant 10 minutes.

(a)

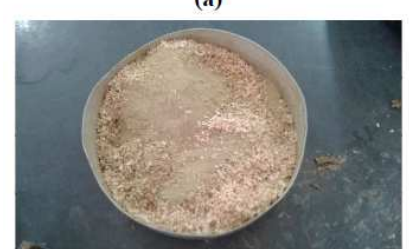

(c)

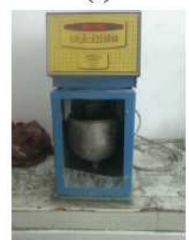

(b)

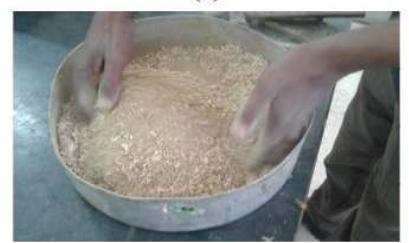

(d)

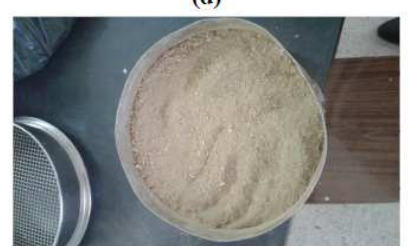

Fig. 2. Malaxage à sec des différents composants

\subsection{Malaxage de la pate}

L'eau est par la suite ajoutée aux autres ingrédients secs. Le tout est mélangé manuellement jusqu'à l'obtention d'une pate homogène. Enfin, le mélange obtenu est malaxé mécaniquement dans un malaxeur électrique pendant 10 minutes jusqu'à l'obtention d'une pate plastique ayant la consistance d'une poudre humide. (a)

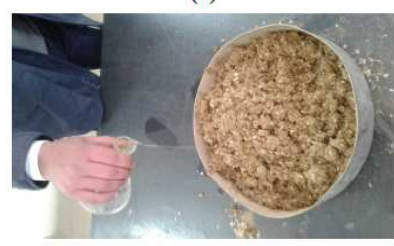

(c)

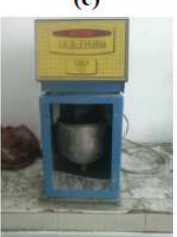

(b)

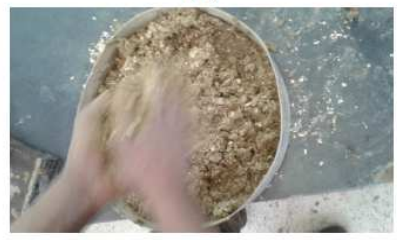

(d)

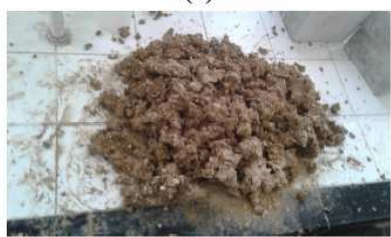

Fig. 3. Malaxage du mélange avec de l'eau

\subsection{Mise en forme :}

Le mélange, ayant la consistance d'une poudre humide, est versé dans un moule métallique.
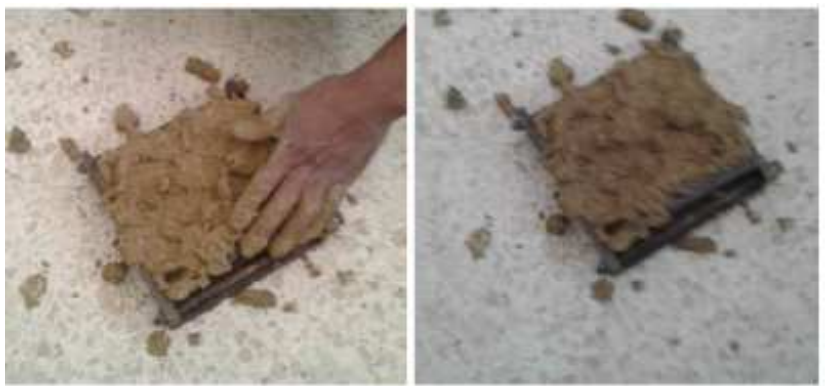

Fig. 4. Moulage des éprouvettes

\subsection{Pressage}

La pate versée dans un moule métallique est compressée sous une pression de $4 \mathrm{MPa}$ à l'aide d'une presse hydraulique de capacité $3000 \mathrm{KN}$. La pression exercée sur les échantillons lors du moulage favorise le tassement des particules d'argile les unes sur les autres, minimisant ainsi les interstices entre les grains et facilite ainsi la formation de liens céramiques assurant une bonne résistance aux échantillons cuits.
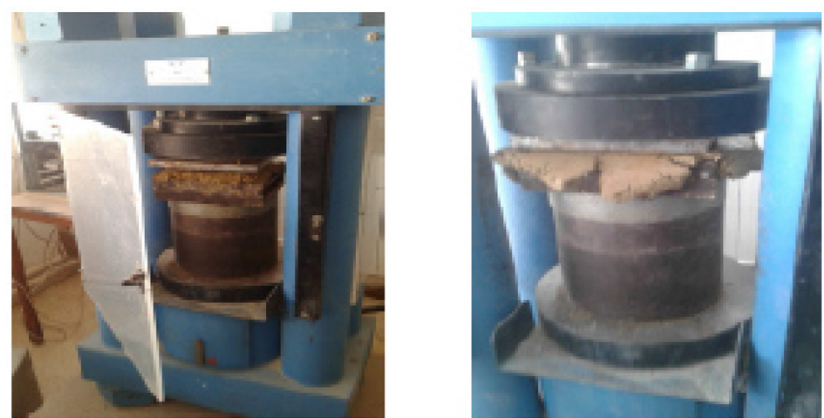

Fig. 5. Pressage des éprouvettes

\subsection{Démoulage}

Les briques sont alors démoulées en dévissant complètement les parois du moule.

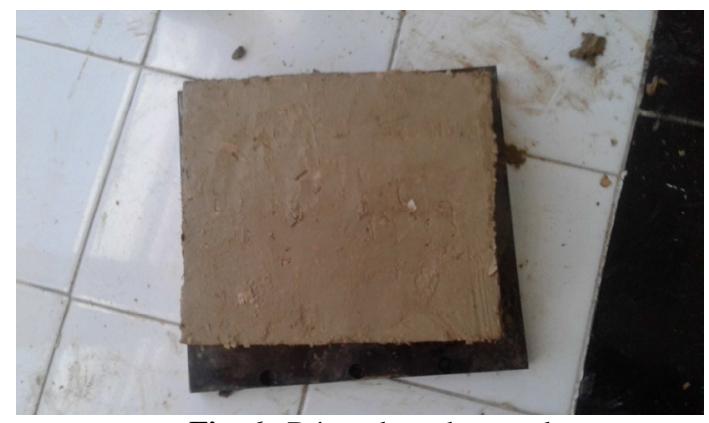

Fig. 6. Démoulage des moules

\subsection{Séchage}

Les briques ont été séchées dans une enceinte climatique. Le séchage est effectué selon le procédé utilisé par la majorité des briqueteries à une température allant de $40^{\circ} \mathrm{C}$ jusqu'à $110^{\circ} \mathrm{C}$ pendant 4 heures : 
Le programme de séchage adéquat est composé des paliers suivants :

$\checkmark 0.5$ heure à une température de $40^{\circ} \mathrm{C}$.

$\checkmark 1.5$ heure à une température de $60^{\circ} \mathrm{C}$.

$\checkmark 1$ heure à une température de $80^{\circ} \mathrm{C}$.

$\checkmark 1$ heure à une température de $110^{\circ} \mathrm{C}$.

\section{Détermination du programme de cuisson}

\subsection{Le programme expérimental}

Cette partie de l'étude a pour objectif d'étudier le comportement des briques lors de la cuisson et ceci pour définir le programme de cuisson convenable pour ces briques afin de produire une brique d'argile poreuse de faible densité dont les propriétés répondent aux exigences spécifiées par les normes des briques.

Plusieurs échantillons, composés de l'argile $+4 \%$ de sciures de bois $+20 \%$ d'eau, ont été fabriqués et cuits à des températures intermédiaires $300,550,850$ et $1050^{\circ} \mathrm{C}$. Ce programme de cuisson de 23 heures est présenté sur la figure 7.

Pour chacune des températures, la disparition progressive de la coloration noire à l'intérieur des échantillons a été notée, de même que les pertes de poids en fonction du temps de cuisson et de la température.

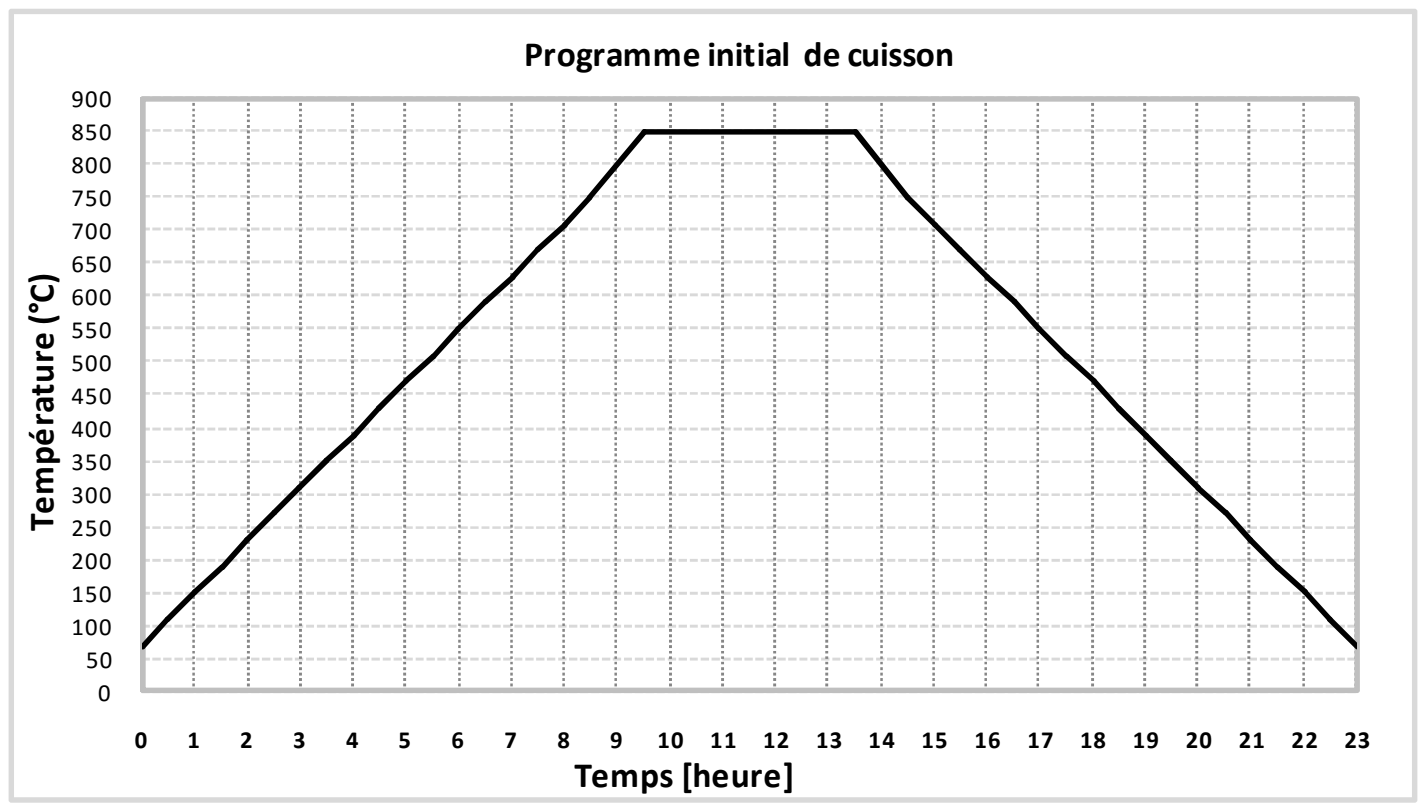

Fig. 7. Le programme de cuisson initial

\subsection{Résultats et discussions}

\subsubsection{Inspections visuelles des échantillons}

L'un des changements, immédiatement décelable visuellement, est la modification de la couleur sous l'action de la température. Les échantillons sont passés d'un gris terne après le séchage, à des tonalités de jaune, d'orange et finalement à des teintes de brun-rouge. Les changements de coloration sont habituellement occasionnés par la présence dans l'argile à l'état brut, d'oxydes ferriques $\left(\mathrm{Fe}_{2} \mathrm{O}_{3}\right)$ qui changent graduellement de couleur sous l'action de la température.

En ce qui concerne l'incinération des sciures de bois prises isolément, une cuisson d'une heure à $300^{\circ} \mathrm{C}$ est suffisante pour que $95 \%$ du poids initial de sciures soit consumé. Sous l'action de la température les sciures se consument et créent des pores à l'intérieur des briques.

Toutefois, cette combustion est incomplète à des températures inférieures à $850^{\circ} \mathrm{C}$. En effet, une teinte noire foncée prédomine, en particulier à la suite de cuisson à des températures de 300 et $400^{\circ} \mathrm{C}$. A 500, 600 et $700^{\circ} \mathrm{C}$, le noir s'estompe à la surface des échantillons et laisse place graduellement à un jaune-brun. Cette couleur noire est vraisemblablement due à la présence de composés de carbone.

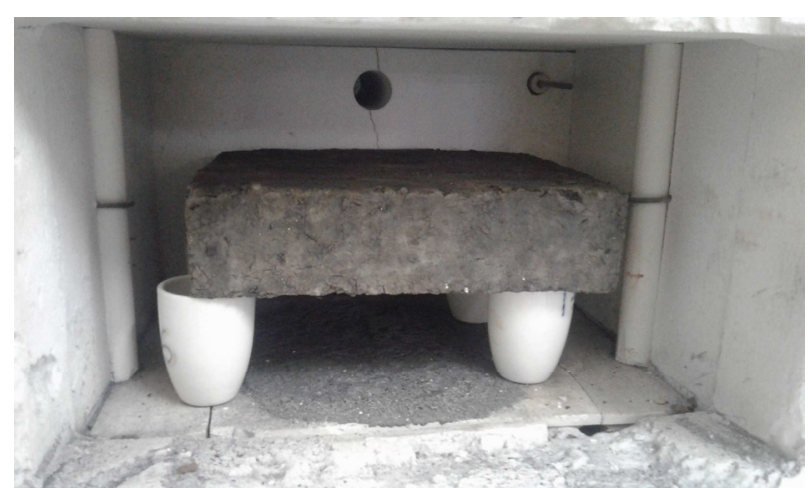

Fig. 8. Début de l'incinération de sciures de bois à $300^{\circ} \mathrm{C}$

Les résultats montrent aussi qu'après une cuisson de 4 heures à $300{ }^{\circ} \mathrm{C}$ la combustion des sciures de bois est quasi-totale. Un examen visuel indique toutefois que des traces résiduelles subsistent à la suite de cuisson à des températures plus élevées. 


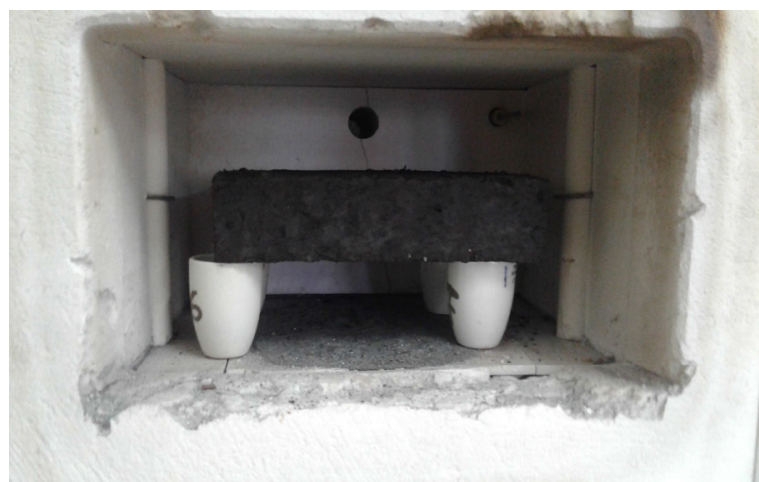

Fig. 9. Incinération de sciures de bois à $300{ }^{\circ} \mathrm{C}$ pendant $4 \mathrm{~h}$

Une inspection visuelle permet de constater que pour un temps de cuisson d'une heure à $550^{\circ} \mathrm{C}$ réduit progressivement la coloration noire des échantillons jusqu'à ce qu'elle disparaisse complètement après 4 heures.

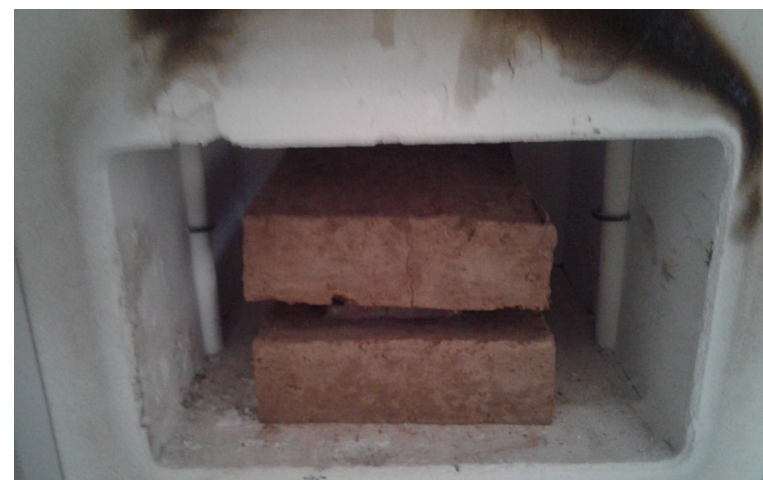

Fig. 10. Etat des briques après 4 heures de cuisson à $550{ }^{\circ} \mathrm{C}$.

Enfin, le temps et la température de cuisson influencent la combustion des sciures. Plus la température est élevée, plus les sciures se consument rapidement. Les températures ont été de 300,550 et $850^{\circ} \mathrm{C}$ avant que la température soit élevée à $1050^{\circ} \mathrm{C}$.

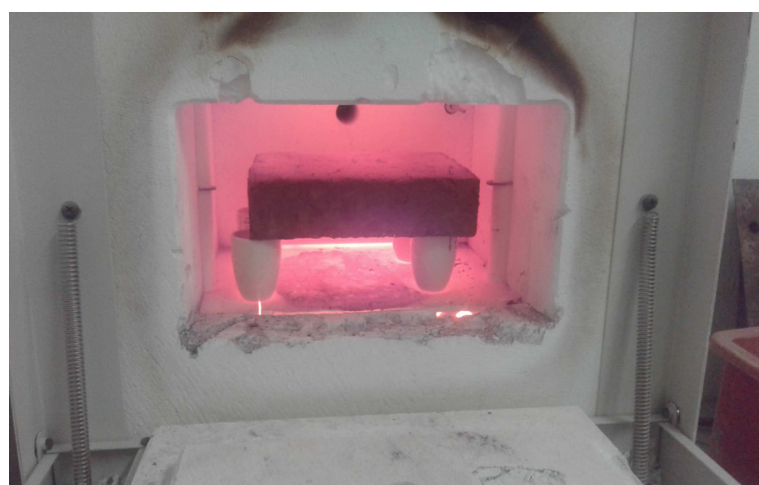

Fig. 11. Cuisson des briques à $850^{\circ} \mathrm{C}$

La figure 12 montre l'expansion d'une brique après une heure de cuisson à $1050^{\circ} \mathrm{C}$. Cette expansion débute, comme telle, à partir de $850^{\circ} \mathrm{C}$ et progresse suite à une cuisson d'une heure à $1050^{\circ} \mathrm{C}$.

En effet, l'augmentation de la température de 850 à $1050^{\circ} \mathrm{C}$ environ, affecte la microstructure de la brique. Les pores, généralement ronds, petits et uniformément répartis à la suite d'une cuisson pendant une heure à $1050^{\circ} \mathrm{C}$ environ, font graduellement place à des pores de plus grosses dimensions et de formes irrégulières. La courbe de la variation de la densité apparente des briques en fonction de la température de cuisson illustre cette progression (Figure 16).

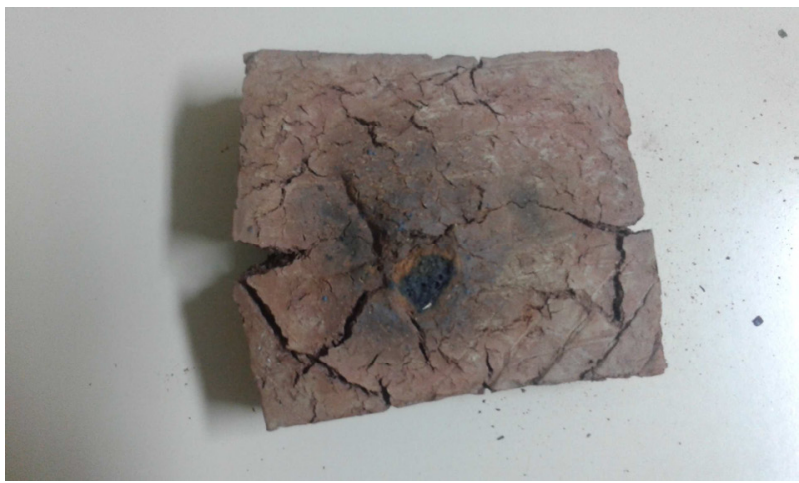

Fig. 12. Expansion d'une brique après $1 \mathrm{~h}$ de cuisson à $1050^{\circ} \mathrm{C}$

Des zones noires, clairement délimitées, ont été décelées à l'endos et au centre des échantillons cuits pendant 2.5 heures à $1050^{\circ} \mathrm{C}$. Ces zones ont une apparence vitreuse et une surface polie et brillante. Elles sont accompagnées sans exceptions d'une déformation des échantillons telle qu'en témoigne la figure 13. Ce phénomène, dénommé "cœurs noirs" .

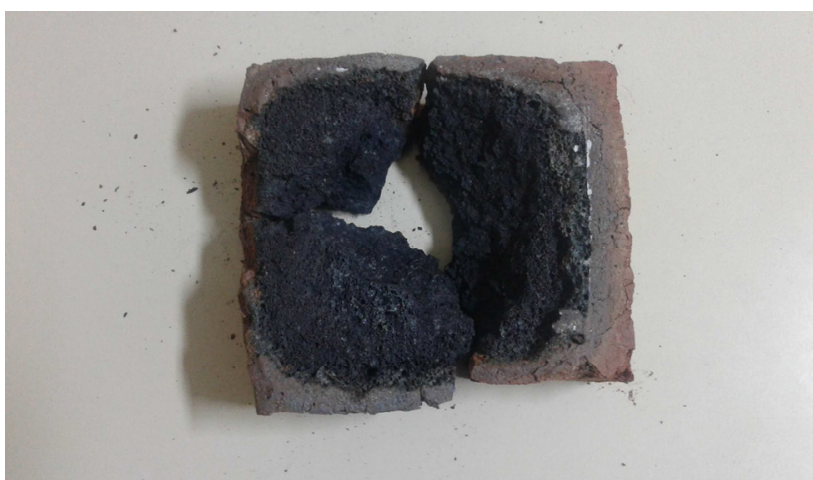

Fig. 13. Cœurs noirs au niveau d'une brique

\subsubsection{Variation de la résistance à la compression en fonction de la température de cuisson}

A partir des courbes présentées sur la figure 15, on peut distinguer deux zones: une zone de croissance de la résistance jusqu'à une température de $850^{\circ} \mathrm{C}$; et une zone de décroissance à partir de $850^{\circ} \mathrm{C}$.

L'augmentation de la résistance est probablement due au fait que durant la cuisson les oxydes ferreux agissent comme fondants et que l'argile ramollie se solidifie durant la période de refroidissement et lie très fortement les particules d'argile entre elles et entrainant une augmentation marquée de la résistance mécanique des échantillons.

La chute de la résistance à partir de $850^{\circ} \mathrm{C}$ est vraisemblablement attribuable à la formation de cœurs noirs qui entrainent une augmentation de la porosité, de gonflements et un craquelage en surface. Ces craques rompent la continuité des liens solides établis, et diminuent la résistance en compression des échantillons. 


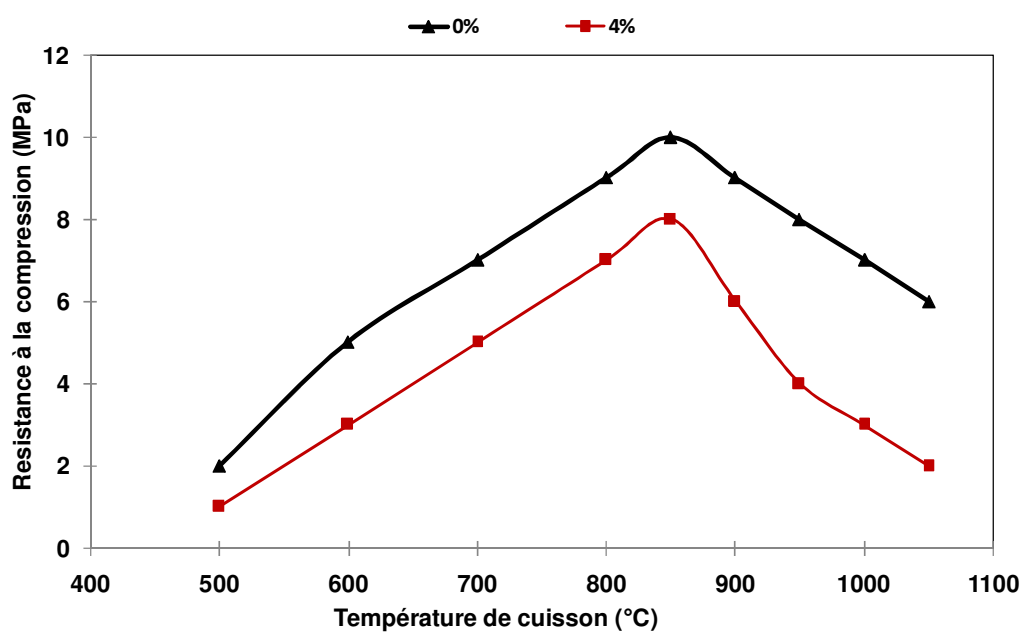

Fig. 15. Variation de la résistance à la compression des briques en fonction de la température de cuisson

\subsubsection{Conclusions et recommandations :}

Suite aux résultats présentés, on peut conclure qu'en variant la durée de cuisson, les meilleurs résultats ont été obtenus après 3.5 heures de cuisson à $850{ }^{\circ} \mathrm{C}$. (Fig. 16).

Finalement, on peut adopter le programme de cuisson présenté sur la figure 17.

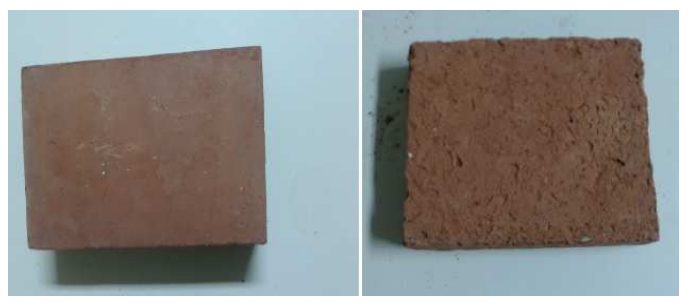

Fig. 16. Etat des briques après 3,5 heures de cuisson à $850^{\circ} \mathrm{C}$ : Brique d'argile et brique d'argile et de sciures de bois

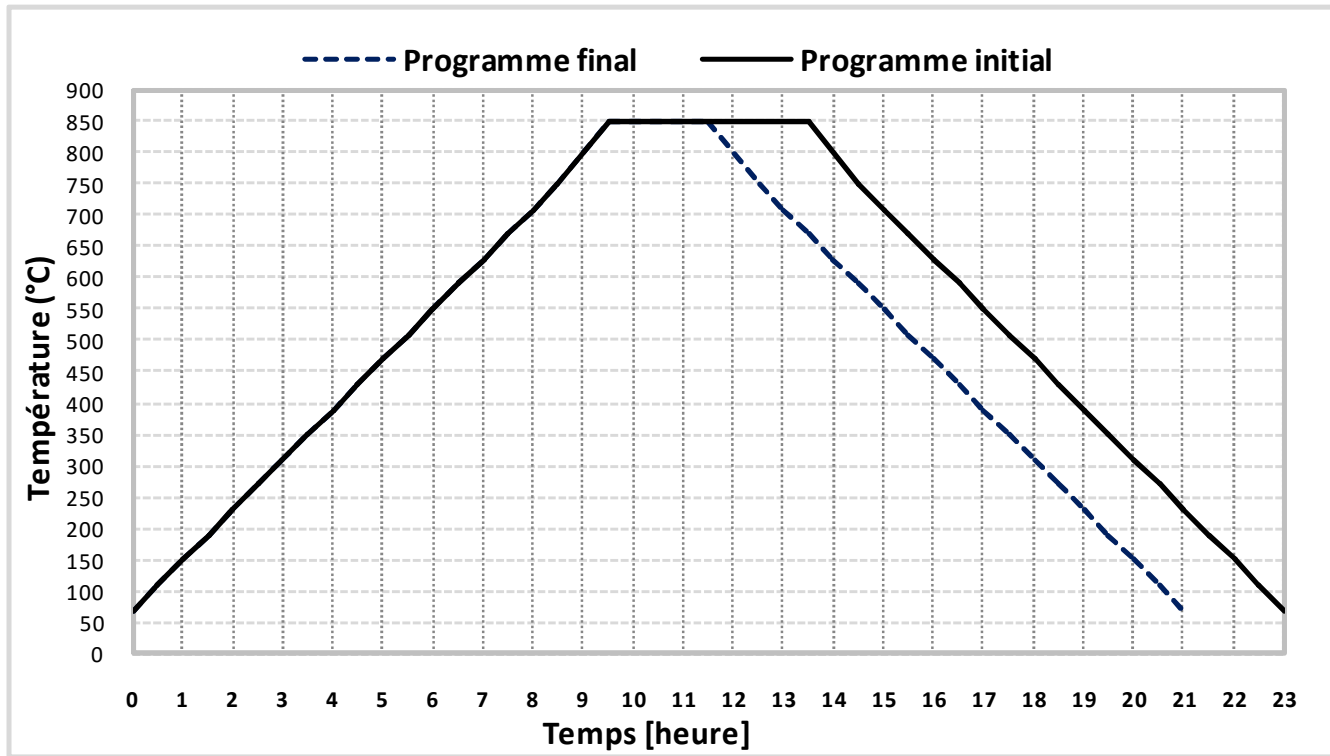

Fig. 17. Le programme de cuisson adopté

\section{Variation de la quantité de sciures}

\subsection{Le programme expérimental}

L'objectif de cette partie de l'étude est d'étudier les modifications des propriétés physiques et mécaniques qu'entraine l'addition d'une quantité variable de sciures de bois à l'argile. Pour aboutir à ces objectifs, 6 séries d'échantillons ont été ainsi préparés à partir des compositions présentés dans le tableau 2.

Tableau 2. Compositions des différents échantillons

\begin{tabular}{|c|c|c|}
\hline Série & Proportion de sciures debois (\%) & Proportion d'eau (\%) \\
\hline S1 & 0 & 15 \\
\hline S2 & 2 & 18 \\
\hline S3 & 4 & 22 \\
\hline S4 & 6 & 25 \\
\hline S5 & 8 & 28 \\
\hline S6 & 10 & 30 \\
\hline
\end{tabular}




\subsection{Résultats et discussions}

\subsubsection{Effet de la quantité de sciures sur la densité des briques}

La variation de la densité de la brique en fonction de ratio des sciures de bois est présentée sur la figure 18 .
D'après cette courbe, on remarque que la densité apparente diminue de façon inversement proportionnelle à la quantité de sciures de bois; passant de $2.09 \mathrm{~g} / \mathrm{cm}^{3}$, pour les échantillons sans sciures, à $1.21 \mathrm{gr} / \mathrm{cm}^{3}$ pour ceux constitués de $10 \%$ de sciures.

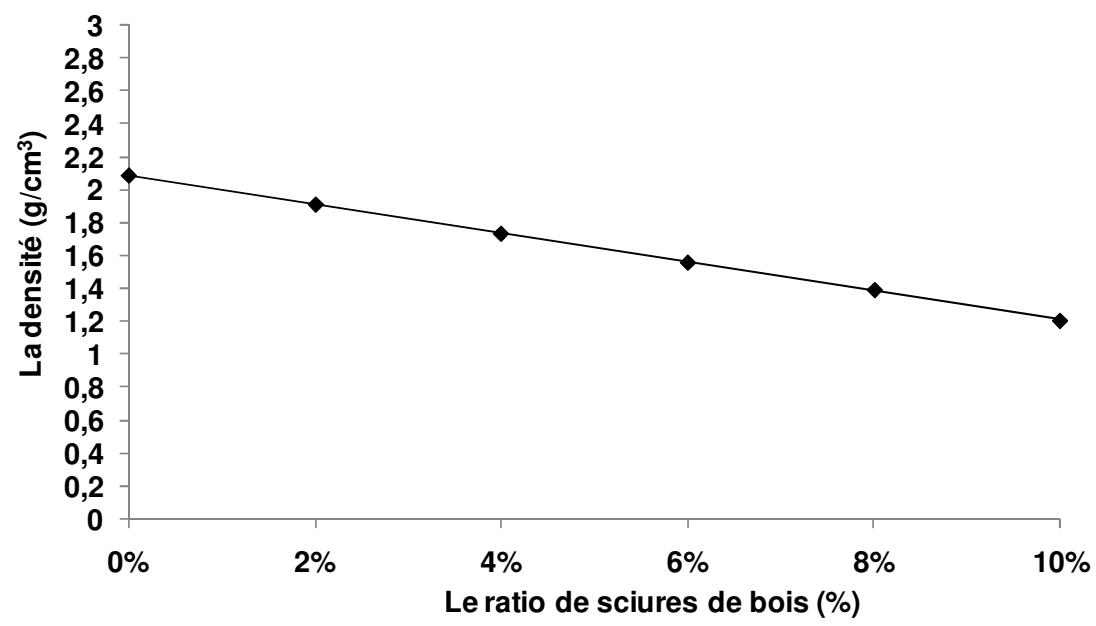

Fig. 18. Variation de la densité des briques en fonction du ratio de sciures de bois

\subsubsection{Effet de la quantité de sciures sur le taux désorption d'eau des brique}

D'après les résultats présentés sur la figure 19 , on remarque que les taux d'absorption de l'eau augmentent de façon linéaire avec le ratio de sciures additionnées. On remarque aussi qu'au delà d'une quantité de sciures de $6 \%$, les pourcentages d'absorption grimpent alors plus rapidement.
L'addition d'une faible quantité de sciures, 2 $\%, 4 \%$ et $6 \%$ crée de petits pores uniformément distribués. Les particules de sciures semblent bien se répartir dans les mélanges et conservent à l'argile cuite sa structure homogène. L'ajout de plus de $6 \%$ de sciures provoque la création de gros pores localisés en certains endroits ou encore la formation de pores allongés. Ces grands pores constituent des passages supplémentaires qui facilitent la pénétration de l'eau ce qui explique les augmentations plus importantes des taux d'absorption.

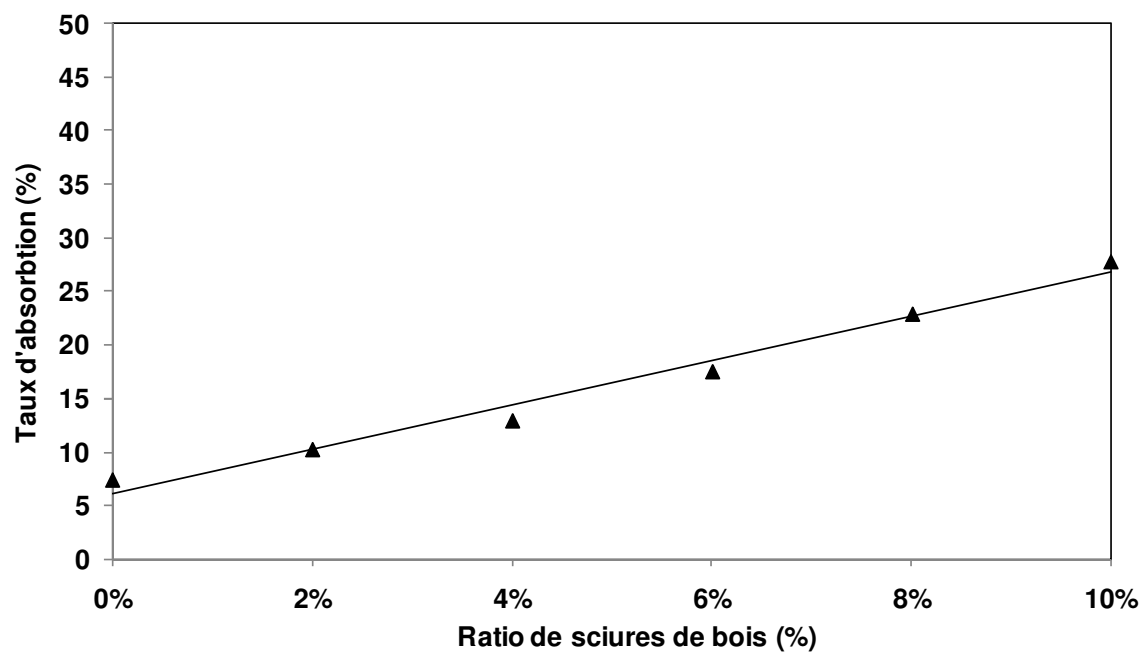

Fig. 19. Variation du taux d'absorption des briques en fonction du ratio de sciures de bois

\subsubsection{Effet de la quantité de sciures sur la résistance en compression des briques}

La figure 20 présente la valeur de la résistance à la compression en fonction du ratio de sciures de bois additionnés à l'argile. A partir de cette figure, on remarque que les valeurs de la résistance en compression chutent très rapidement en fonction de la quantité de sciures ajoutée aux mélanges. 


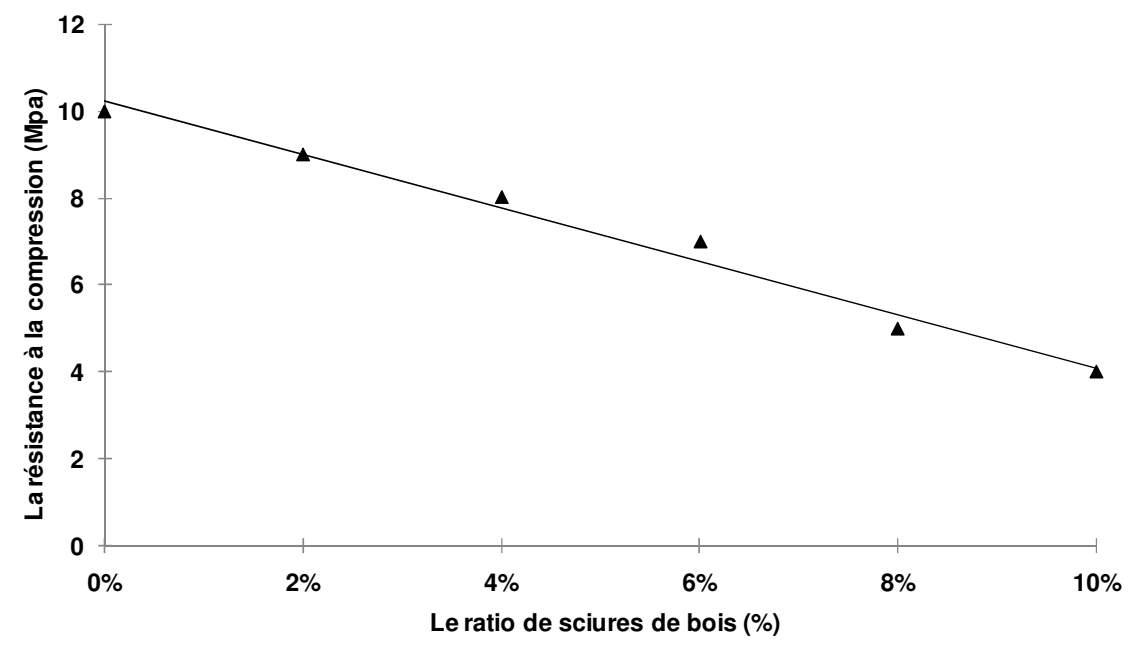

Fig. 20. Variation de la résistance à la compression des briques en fonction du ratio de sciures de bois

La diminution de la résistance lorsque on ajoute de sciures de bois à l'argile peut être expliquée par le fait que plus la quantité de sciures augmente, plus les grains d'argile sont distanciés les uns des autres, et plus la consolidation de ces grains est difficile au moment de la cuisson. En effet, les échantillons cuits offrent alors l'apparence d'une masse de particules non-cimentées qui s'effrite d'ailleurs facilement. Ce phénomène est particulièrement marqué lorsque l'ajout de sciures est plus que $6 \%$ par poids.

\section{Conclusion}

L'objectif principal de cette étude est de produire des briques d'argile plus légères que celles qui sont actuellement sur le marché, en ajoutant des sciures de bois aux mélanges d'argile. Il a été démontré inclusivement que l'ajout de sciures modifie de façon marquée les propriétés physiques et mécaniques des échantillons cuits et que pour optimiser ces propriétés il faut modifier de façon sensible les procédés de fabrication et de cuisson habituels des briques d'argile.

Le processus de fabrication, de séchage et de cuisson était adapté au nouveau produit pour obtenir un abaissement de la densité apparente des produits cuits à base de sciures de bois, tout en minimisant l'accroissement de taux d'absorption et en limitant le plus possible la diminution la résistance en compression.

Une température maximale de cuisson de $850^{\circ} \mathrm{C}$ a été considérée comme optimale, cette température étant voisine à celle ou la même que pour la température de cuisson des briques à base d'argile.

L'addition d'une quantité croissante de sciures tend donc à modifier sensiblement les propriétés physiques et mécaniques des échantillons cuits. La présence de sciures réduit le nombre de connections entre les grains d'argile et produit un matériau plus léger, plus absorbant et moins résistant.

Enfin, les résultats obtenus dans ce projet montrent que la confection des briques à base des sciures de bois est possible. Les résultats des essais mécaniques confortent cette possibilité d'utilisation.

\section{Références}

1. Al-Marahleh, G., 2005. Production of light weight ceramics tiles from local materials. Am. J. Appl. Sci. 2 (2), 778-783.

2. Demir, I., 2005. An investigation on the production of construction brick with processed waste tea. Build. Environ. 41, 1274-1278.

3. Demir, I., 2008. Effect of organic residues addition on the technological properties of clay bricks. Waste Manage. 28 (3), 622-627.

4. Dominguez, E.A., Ullmann, R., 1996. 'Ecological bricks' made with clays and steel dust pollutants. Appl. Clay Sci. 11 (2), 237-249.

5. Dondi, M., Marsigli, M., Fabbri, B., 1997a. Recycling of industrial and urban wastes in brick production: a review. Tile Brick Int. 13 (4), 218-225.

6. Dondi, M., Marsigli, M., Fabbri, B., 1997b. Recycling of industrial and urban wastes in brick production - a review (Part 2). Tile Brick Int. 13 (3), 218-225.

7. Dondi, M., Marsigli, M., Venturi, I., 1999. Microstructure and mechanical properties of clay bricks: comparison between fast firing and traditional firing. Br. Ceram. Trans. 98 (1), 12-18.

8. Pavlola, L., 1996. Use of industrial waste in brick manufacture. Tile Brick Int. 12 (3), 224-225.

9. Cusido, J.A., Cremades, L.V., Gonzalez, M., 2003. Gaseous emissions from ceramics manufactured with urban sewage sludge during firing processes. Waste Manage. 23 (3), 273-280.

10. M. Dondi, M. Marsigli, B. Fabbri, Recycling of industrial and urban wastes in brick production, Tile and Brick International 13 (3) (1997) 218- 225.

11. V. Ducman, T. Kopar, The influence of different waste additions to clay- product mixtures, Materials and Technology 41 (6) (2007) 289-293. 